\title{
CORRESPONDENCE \\ What should a paediatrician know about qualitative knowledge synthesis?
}

\author{
Pediatric Research (2022) 91:1308-1310; https://doi.org/10.1038/ \\ s41390-021-01424-7
}

\begin{abstract}
INTRODUCTION
Knowledge synthesis can be defined as "the contextualization and integration of research findings of individual research studies within the larger body of knowledge on the topic". ${ }^{1}$ The synthesis can be done using quantitative and/or qualitative approaches. The development of the qualitative knowledge synthesis methodologies and the rate of their use in the field of health care is growing significantly. ${ }^{2}$ That growth is reflected in the substantial increase in the qualitative research literature production. Already in 2015 the Joanna Briggs Institute, an organisation concerned with evidence-based improvement in health-care practices and health outcomes, cautioned that the field of qualitative synthesis entered into the phase, where comparative evaluation research has become necessary. ${ }^{3}$
\end{abstract}

\section{METHOD}

To detail the status of qualitative synthesis in paediatrics, we designed our bibliometric study ${ }^{4}$ not only to analyse its use but also to enable us to reflect on what we believe a paediatrician should know about qualitative synthesis and why. Our bibliometric study was performed for the period 2016-2020 on the corpus of publications harvested from the Web of Science-All Databases using the search string $T S=\left(\left(\right.\right.$ "meta ${ }^{*}$ " OR "meta- ${ }^{*}$ " OR "* review" OR "** Synthesis") AND qualitative*) AND SU = paediatrics. Bibliometric analysis was performed using the VOSViewer software. $^{5}$

\section{RESULTS AND DISCUSSION}

Our study revealed that despite the high number of paediatric publications dealing with knowledge synthesis (over 72,000), only $3.2 \%$ of them report using qualitative approaches. However, it is also the fact that the number of qualitative synthesis publications increased by $>42 \%$ in the past 5 years.

As a scholarship field, qualitative knowledge synthesis offers a wide spectrum of approaches, most of them founded on a solid theoretical basis. Discussion about their use, goodness of fit for purpose or process steps can be also found in various research publications. Our bibliometric analysis showed that qualitative synthesis in paediatrics is mostly performed by approaches that have roots in quantitative analysis, namely Systematic reviews and Meta-analysis. Among approaches that were developed specifically for qualitative synthesis, Meta synthesis, Narrative Synthesis, Meta-Aggregation and Meta ethnography are most popular. On rare occasions also the Realist synthesis was employed.

Qualitative systematic reviews ${ }^{3}$ should answer complex questions related to subjective perspectives, for example, concerning meanings/beliefs young patients attribute to their experiences (e.g. pain, chronic fatigue or maltreatment) or Weltanschauung behind certain behaviours like "Vaccination is good for my child" or "breastfeeding in neonatal units has positive effects". In particular, questions in qualitative synthesis (not limited only to qualitative systematic reviews) should begin with "what is it like" (e.g. "not being in school for so long because of COVID-19" or "having Type 1 diabetes"), or "what is the experience of" (e.g. "being hospitalised for paediatric long-term condition" or "being part of parenting programmes for childhood behaviour problems") and "why do children" (e.g. "play computer games" or "don't participate in leisure-time physical activity interventions") are often used to demonstrate the areas that qualitative synthesis addresses.

Qualitative meta-analysis ${ }^{6}$ has a similar objective to quantitative meta-analysis, namely to assess a field of study beyond one particular study. It aims to conduct a rigorous secondary qualitative analysis of primary qualitative findings investigating the same general research topic, with the objective to obtain a comprehensive description of a phenomenon being researched and to identify an interpretation of findings that is more substantive than those resulting from individual investigations. On the other hand, meta-synthesis ${ }^{7}$ also attempts to integrate results from different qualitative studies; however, in an interpretive, rather than in an aggregating manner.

Narrative synthesis ${ }^{8}$ is an approach to evidence synthesis based on a formal analytical process (preliminary synthesis of findings, exploring relationships in the data and assessing the robustness of synthesised findings) aiming to generate new insights or knowledge by being systematic and transparent.

Meta-ethnography ${ }^{9}$ is an interpretive approach focussing on constructing new interpretations from existing qualitative literature on the mid-level theoretical level. New interpretations are expressed through new models, theories and concepts. Metaaggregation is another integrative synthesis approach, however, not aiming at re-interpretation of findings, but on a practice level theory, appropriate to the information and knowledge needs for real-world problem-solving.

Realist synthesis ${ }^{10}$ aims to extend/complement classical systematic reviews, normally concerned with "simpler" interventions (i.e. clinical treatments or therapies) in synthesising research evidence on complex social interventions. They perform an explanatory analysis of how and why complex interventions may or may not work in particular social/cultural settings.

The qualitative part (Fig. 1) of our bibliometric analysis revealed that:

- Qualitative systematic reviews and meta-analysis studies are concerned with younger paediatric populations, covering the period from infancy to early childhood. The research is performed in the context of chronic diseases, outcome research, physical activity, clinical trials, obesity, breastfeeding, global health, nutrition, treatment/therapy, neonatal care, intensive care, malnutrition and radiology. The main 


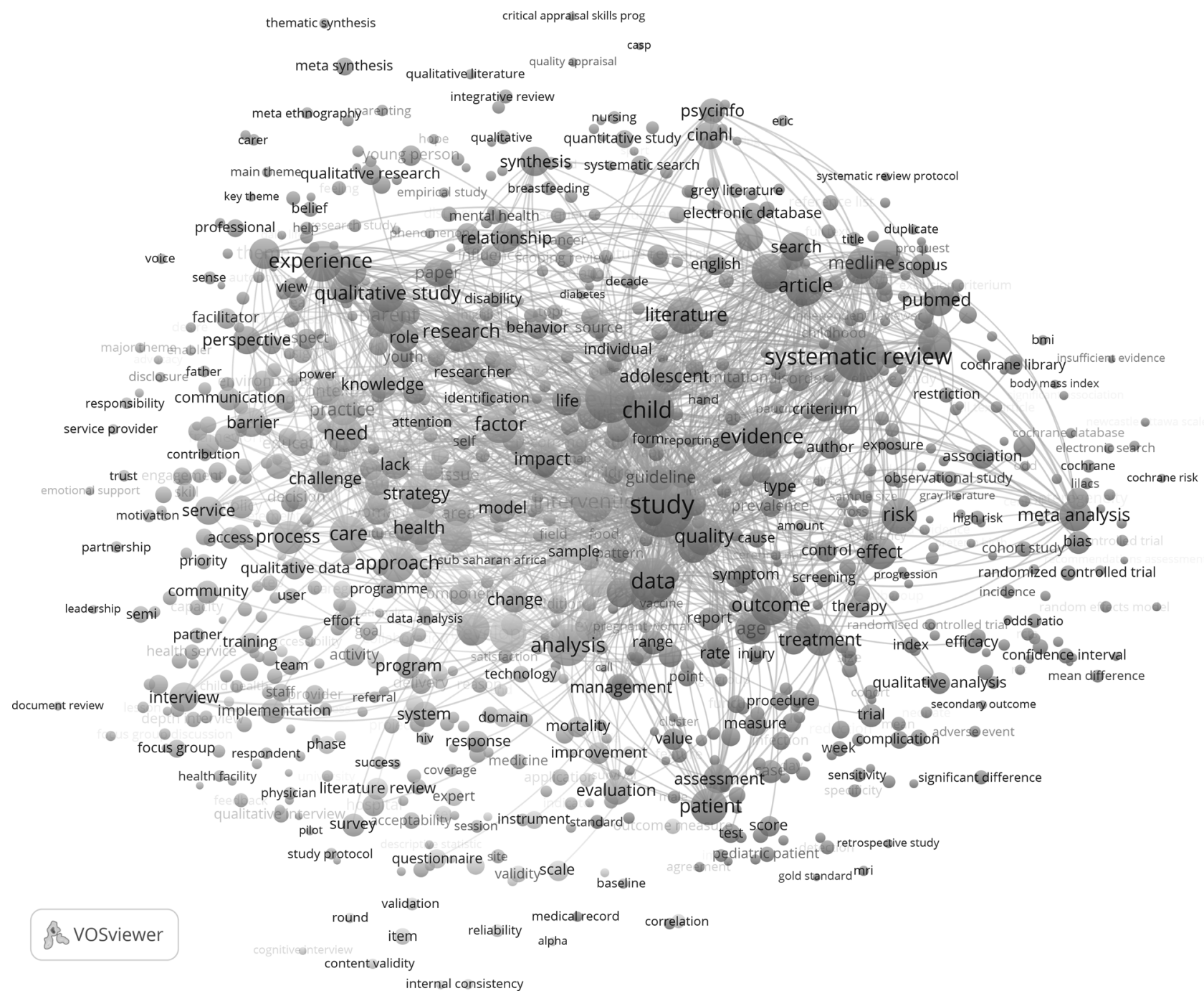

Fig. 1 Bibliometric term landscape of qualitative synthesis in paediatrics. Red cluster is concerned with systematic reviews and metaanalysis; blue cluster with meta-synthesis, meta-ethnography, and narrative and realist synthesis; and blue and yellow clusters with methodological and validation aspects of qualitative analysis.

bibliographic databases used are Web of Science, Scopus, PubMed, Google Scholar, Embase and Cochrane.

- Meta-ethnography, meta-aggregation and meta-synthesis studies are concerned mainly with young adults and are performed in the thematic context of parental experience, mental health, nursing, suicide, well-being, disability, violence, self-management of care, child maltreatment, paediatric health professionals behaviour and problems, emotional support. The main databases used are CINAHL and Science direct.

- Narrative synthesis-based research is mainly concerned with childhood cancer, intellectual disability and various interventional studies.

- Realist synthesis studies are primarily related to mobile health, health technology, symptom management and social media use in paediatric health care.

The quantitative aspect of our bibliometrics analysis may provide some guidance to help paediatricians who are interested in not only reading but also performing and possibly publishing qualitative synthesis studies in (1) locating possible research partners, (2) identifying journals for publishing results of their studies or (3) finding promising funding agencies. Most productive countries producing $>80 \%$ of qualitative synthesis publications are United States of America (USA), United Kingdom (UK), Canada, Australia and Germany. University of London (UK), University of Toronto (Canada), Harvard University (USA), University College of London (UK) and Johns Hopkins University (USA) are the most productive institutions. BMJ Open, PLoS One, BMC Public Health, International Journal of Environmental research and Public Health and BMC Health Service Research are the most prolific journals. Most qualitative synthesis studies are funded by United States Department of Health and Human Services, USA National Institutes of Health, National Health and Medica Research Council of Australia, USA National Institute for Health Research and Eunice Kennedy Shriver National Institute of Child Health Human Development.

\section{CONCLUSION}

Qualitative knowledge synthesis is a fast-growing field. As reflected from our bibliometric analysis, it is used in paediatrics with a growing rate and in different contexts; nevertheless, its ratio toward quantitative synthesis should become more balanced. Based on our findings, we believe that qualitative synthesis may help answer many important questions related to 
1310

children and adolescents' health or well-being in a more accurate way than by relying on the results of single studies.

\section{AUTHOR CONTRIBUTIONS}

Substantial contributions to conception and design, acquisition of data, or analysis and interpretation of data: P.K., H.B.V. and J.Z. Drafting the article or revising it critically for important intellectual content: H.B., J.Z. and P.K. Final approval of the version to be published J.Z., H.B. and P.K.

\section{ADDITIONAL INFORMATION}

Competing interests: The authors declare no competing interests.

Publisher's note Springer Nature remains neutral with regard to jurisdictional claims in published maps and institutional affiliations.

Peter Kokol ${ }^{1}$, Jernej Završnik ${ }^{2,3,4,5}$ and Helena Blažun Vošner ${ }^{2,3,6}$ ${ }^{1}$ Faculty of Electrical Engineering and Computer Science, University of Maribor, Maribor, Slovenia; ${ }^{2}$ Community Healthcare Centre Dr. Adolf

Drolc Maribor, Maribor, Slovenia; ${ }^{3}$ Alma Mater Europaea-ECM, Koper, Slovenia; ${ }^{4}$ Science and Research Center Koper, Koper, Slovenia; ${ }^{5}$ Faculty of Natural Sciences and Mathematics, University of Maribor, Maribor, Slovenia and ${ }^{6}$ Faculty of Health and Social Sciences Slovenj Gradec, Slovenj Gradec, Slovenia Correspondence: Peter Kokol (peter.kokol@um.si)

\section{REFERENCES}

1. Government of Canada Cl of HR. A guide to knowledge synthesis-CIHR. https:// cihr-irsc.gc.ca/e/41382.html (2010).

2. Sattar, R., Lawton, R., Panagioti, M. \& Johnson, J. Meta-ethnography in healthcare research: a guide to using a meta-ethnographic approach for literature synthesis. BMC Health Serv. Res. 21 (2021).

3. Lockwood, C. Qualitative synthesis: the forgotten knowledge needs of end user. www.nursingcenter.com/journalarticle?Article_ID $=3468383 \&$ Journal_ID $=$ 3425880\&Issue_ID=3468382 (2021).

4. Kokol, P., Blažun Vošner, H. \& Završnik, J. Application of bibliometrics in medicine: a historical bibliometrics analysis. Health Infom. Libr. J. https://doi.org/10.1111/ hir.12295 (2020).

5. van Eck, N. J. \& Waltman L. in Measuring Scholarly Impact: Methods and Practice (eds Ding, Y., Rousseau, R. \& Wolfram, D.) 285-320 (Springer International Publishing, 2014).

6. Levitt, H. M. How to conduct a qualitative meta-analysis: tailoring methods to enhance methodological integrity. Psychother. Res. 28, 367-378 (2018).

7. Sandelowski, M., Docherty, S. \& Emden, C. Qualitative metasynthesis: issues and techniques. Res. Nurs. Health 20, 365-371 (1997).

8. Gallo, K. P., Hill, L. C., Hoagwood, K. E. \& Olin, S. S. A narrative synthesis of the components of and evidence for patient- and family-centered care. Clin. Pediatr. 55, 333-346 (2016).

9. Noblit, G. W. \& Hare R. D. Meta-Ethnography: Synthesizing Qualitative Studies (Sage, 1988).

10. Greenhalgh, T., Harvey, G. \& Walshe K. Realist synthesis: an introduction Ray Pawson. https://www.researchgate.net/publication/228855827_Realist_Synthesis_ An Introduction\#fullTextFileContent (2004). 